\title{
GCU
}

Glasgow Caledonian

University

University for the Common Good

\section{A systematic review of interventions to increase physical activity among South Asian adults}

Horne, M.; Tierney, S.; Henderson, S.; Wearden, A.; Skelton, D.A.

Published in:

Public Health

DOI:

10.1016/j.puhe.2018.05.009

Publication date:

2018

Document Version

Author accepted manuscript

Link to publication in ResearchOnline

Citation for published version (Harvard):

Horne, M, Tierney, S, Henderson, S, Wearden, A \& Skelton, DA 2018, 'A systematic review of interventions to increase physical activity among South Asian adults', Public Health, vol. 162, pp. 71-81.

https://doi.org/10.1016/j.puhe.2018.05.009

\section{General rights}

Copyright and moral rights for the publications made accessible in the public portal are retained by the authors and/or other copyright owners and it is a condition of accessing publications that users recognise and abide by the legal requirements associated with these rights.

Take down policy

If you believe that this document breaches copyright please view our takedown policy at https://edshare.gcu.ac.uk/id/eprint/5179 for details of how to contact us. 


\section{A systematic review of interventions to increase physical activity among South Asian adults.}

Dr Maria Horne ${ }^{1^{\star}}$, Dr Stephanie Tierney², Associate Professor Saras Henderson ${ }^{3}$, Professor A Wearden ${ }^{4}$ and Professor DA Skelton ${ }^{5}$

1 School of Healthcare, University of Leeds, Leeds, LS2 9JT. email: M.Horne@leeds.ac.uk

2 Nuffield Department of Primary Care Health Sciences, University of Oxford, Oxford OX2 6GG email stephanie.tierney@phc.ox.ac.uk

${ }^{3}$ School of Nursing and Midwifery, Griffith University, Menzies Health Institute, Queensland, Australia email: s.henderson@griffith.edu.au

${ }^{4}$ School of Psychological Sciences, University of Manchester, Coupland Building, Oxford Road, Manchester, M13 9PL email: alison.wearden@manchester.ac.uk

${ }^{5}$ School of Health and Social Care, Glasgow Caledonian University, Glasgow, Scotland G4 OHB. email: dawn.skelton@gcal.ac.uk

*Corresponding author: Dr Maria Horne Email: M.Horne@leeds.ac.uk

Word count: 3062 words 
A systematic review of interventions to increase physical activity levels among South Asian adults.

\begin{abstract}
Objective: To: (i) identify interventions aimed at increasing physical activity (PA) levels among South Asian (SA) adults, and (ii) identify the specific changes in the content and delivery mode of interventions designed to increase PA levels among SA people aged 18+.
\end{abstract}

Design: A systematic review of quantitative studies.

Data synthesis: Extracted data were synthesised using a narrative approach.

Data sources: Multiple electronic databases were searched - ASSIA, CINHAL, EMBASE, Medline, SPORTDiscus and PsychINFO. Included papers met the following criteria: 1) population: community dwelling SA adults, aged 18 years and older 2) outcome: reporting determinants of PA, exercise, or combination of the two measured objectively or using self-report. The search was restricted to English language articles published up to 31st January 2017.

Results: 15 trials/programmes (16 papers) met the review criteria. The findings show that involving the target community in developing culturally appropriate interventions appears to be important in their acceptability, delivery and take-up. Using community-based participation in intervention planning, evaluation and research appears to produce culturally and linguistically tailored interventions that address core values, attitudes, beliefs and norms and encourage participation in PA. Further, the use of community health workers and underpinning the interventions with a psychological theory show promise in increasing PA uptake.

Conclusions: This systematic review suggests that making cultural adaptations to PA interventions shows promise, but the evidence base presented currently is not strong. This does not mean that adopting such an approach is ineffective, but that the evidence base is currently lacking.

Keywords: South Asian; Prevention; Health promotion; Behaviour change; Physical Activity Interventions; Cultural adaptations. 


\section{Background}

Physical activity (PA) can reduce the risk of developing major chronic diseases by up to $50 \%$ and the risk of premature death by $20-30 \%[1,2]$. However, $60 \%$ of the world's population do not achieve the minimum PA recommendations $[1,2]$. Minority groups are less physically active than the Western population as a whole [3] and suffer disproportionately higher rates of certain health conditions $[4,5]$. Specifically, South Asian (SA) people (those originating from the countries of India, Pakistan, Bangladesh and Sri Lanka) are less likely to exercise compared to their Caucasian peers, but experience greater levels of heart-disease and type 2-diabetes mellitus (DM) $[5,6]$. Additionally, SAs may have to exercise more than their white European counterparts to achieve the same levels of fitness to reduce their risk of DM [7].

Meeting the health needs of minority groups is a public health challenge, particularly in early intervention, prevention and reducing health inequalities [8-11]. Although some progress has been made in advancing our understanding of adapting behavioral interventions for minority groups [12], little evidence exists on effective interventions tailored to their needs [13,14]. Minority groups are generally treated as homogenous, leading to inappropriate generalisations and potentially unsuitable interventions [15].

Interventions that are effective in the general population are likely to prove effective among minority groups if appropriately tailored $[13,15]$. Cultural adaptations are modifications made to programmes so they reflect a cultural group's traditional world view and lifestyle and address within group differences [16]. For example, matching intervention messages to observable, surface characteristics of culture, i.e. language, dress [12] or by targeting deeper structures of culture, such as explanatory models about the causes of health and illness [17] and unique barriers/facilitators to changing behaviour [18].

If interventions are to be successful they must respond to cultural, religious and economic issues to address health needs [19]. Therefore, segmenting populations into subgroups by their cultural characteristics can augment receptivity to, acceptance of, and salience of health messages [18]. It is crucial that healthcare professionals understand the needs of diverse groups if they are to adapt evidence-based interventions [20] and tailor information and support so it is culturally acceptable and appropriate $[9,13.21,22]$ to effectively promote behaviour change $[10,21,23,24]$. 
Recent systematic reviews have focussed on identifying what is known about PA levels and sedentary time among SA women [25]; levels of PA among SA adults residing in South Asia [26]; assessment of evidence for effectiveness of primary care based PA and dietary interventions in SA populations [27]; diet and PA interventions to prevent or treat obesity in SA children and adults [28]. No available reviews have focussed specifically on changes made to interventions to increase PA levels among SA adults.

\section{Aim}

The aim of our systematic review was to: (i) identify interventions aimed at increasing PA levels among SA adults, and (ii) identify the specific changes in the content and delivery mode of interventions designed to increase PA levels among SA people aged 18+.

\section{Methods}

Design

A structured systematic literature search was performed in January 2017 using established standards $[29,30]$, with PRISMA guidelines used to inform conduct and reporting [31]. Search terms and strategy are presented in Box 1. The selection, inclusion and exclusion criteria are outlined in Box 2. Initial pilot searches found few studies that focused specifically on PA as the main outcome. Therefore, in addition to studies that specifically focussed on PA uptake and adherence as a primary outcome, we included studies that included PA uptake and adherence as a secondary outcome.

\section{Box 1: Search terms and search strategy}

Search terms

Search terms were developed under the headings 'South Asian' and 'Intervention' and 'uptake or maintenance'. 'Truncation ( $\left.{ }^{\star}\right)$ was employed where variations of a search term existed. Broad search terms were used to ensure that all studies meeting the inclusion criteria were captured. Keywords included combinations of "physical activity", "physical exercise", "exercise", "sport", "physical training" and "Indian" or "Pakistani" or "Bangladeshi" or "South Asian". A copy of the search terms used is available upon request from the first author.

Search strategy 
Full holdings of the following six electronic databases were searched for references from first publication to 31st January 2017: ASSIA, Cumulative Index to Nursing and Allied Health Literature (CINAHL), EMBASE, Medline, PsycINFO and SPORTDiscus. A limit was not placed on the years searched for each database to enable the broadest capture of papers for comparison; however no records published prior to 1991 were returned from searches. Additional articles were identified by searching the references of included articles as well as systematic reviews, which were not included in our review.

\section{Box 2: Selection, inclusion and exclusion criteria}

Titles and abstracts located through the search process were screened by two reviewers (MH and ST) to identify relevant literature, which were then included for further assessment if they met the following criteria:

(1) participants were SA adults aged 18 years or older

(2) the study included assessment of an intervention or programme that included some attention to addressing PA

(3) randomised controlled trials, controlled before-and-after experimental, pre-test post-test, quasiexperimental.

(4) an outcome measure of PA was reported (objective or self-report measure)

(5) provided a measure of uptake and/or adherence as the proportion of participants who fully or partially completed the intervention

(6) published in English language, in a peer-reviewed journal.

Papers were excluded if they met any of the following criteria:

(1) studies without adult data

(2) studies focusing on other minority groups (not SA).

Systematic reviews were excluded to avoid overlap as we wanted to review primary data only; however references of retrieved systematic reviews were searched for any further references.

Where there were any disagreements regarding the inclusion of a paper, consensus was reached through discussion amongst the researchers. 
Inclusion criteria

Types of intervention

Any intervention including PA or exercise at home or in the community were included. PA and exercise were defined as being general or structured movement of the body that would increase energy expenditure [32].

Types of studies

Preliminary pilot searches found few randomised controlled trials (RCTs) of PA interventions for SA people aged 18+ years. Therefore, we decided to include all relevant study designs (eg. RCT, non-RCT, controlled before-after studies, pre-test post-test) to draw from as wide an evidence base as possible.

Quality assessment \& data abstraction

Quality of study design, including methods selection, identification of biases, appropriate use of statistical methods, and clarity of reporting was assessed using a validated checklist developed from the Scottish Intercollegiate Guidelines Network [33]. Study eligibility was confirmed by two researchers. Quality assessment and data extraction were performed by the first author (MH) and confirmed by one of four research team members.

Extracted data included socio-demographic characteristics; country of origin; sample size; intervention/control description; intervention outcomes - PA uptake/adherence, and intervention effects. Uptake and adherence were defined as those who initially participate in a PA intervention and those who continued participation in the intervention on follow-up, respectively. Long-term adherence was defined as 6-months or more [34]. Analyses of the retrieved papers were carried out between MarchMay 2017.

\section{Synthesis}

Findings from the included studies are described numerically and textually to provide a summary of evidence on interventions aiming to increase PA uptake and adherence among SAs aged $18^{+}$, to assess what cultural adaptations were made to take into account a person's ethnic background and their impact on PA uptake and adherence. Due to the small number of included studies and 
heterogeneity in intervention type, assessment and outcome measures, meta-analysis was not possible, so a narrative synthesis was performed [35].

\section{Results}

The study selection process is illustrated in Figure 1. Searches returned 4855 hits (excluding duplicates), of which16 papers, reporting on fifteen trials/programmes [36-51] met the inclusion criteria. Key characteristics and quality ratings are outlined in Table 1; cultural adaptations and design theory in Table 2. 
Figure 1: PRISMA flow diagram of study selection

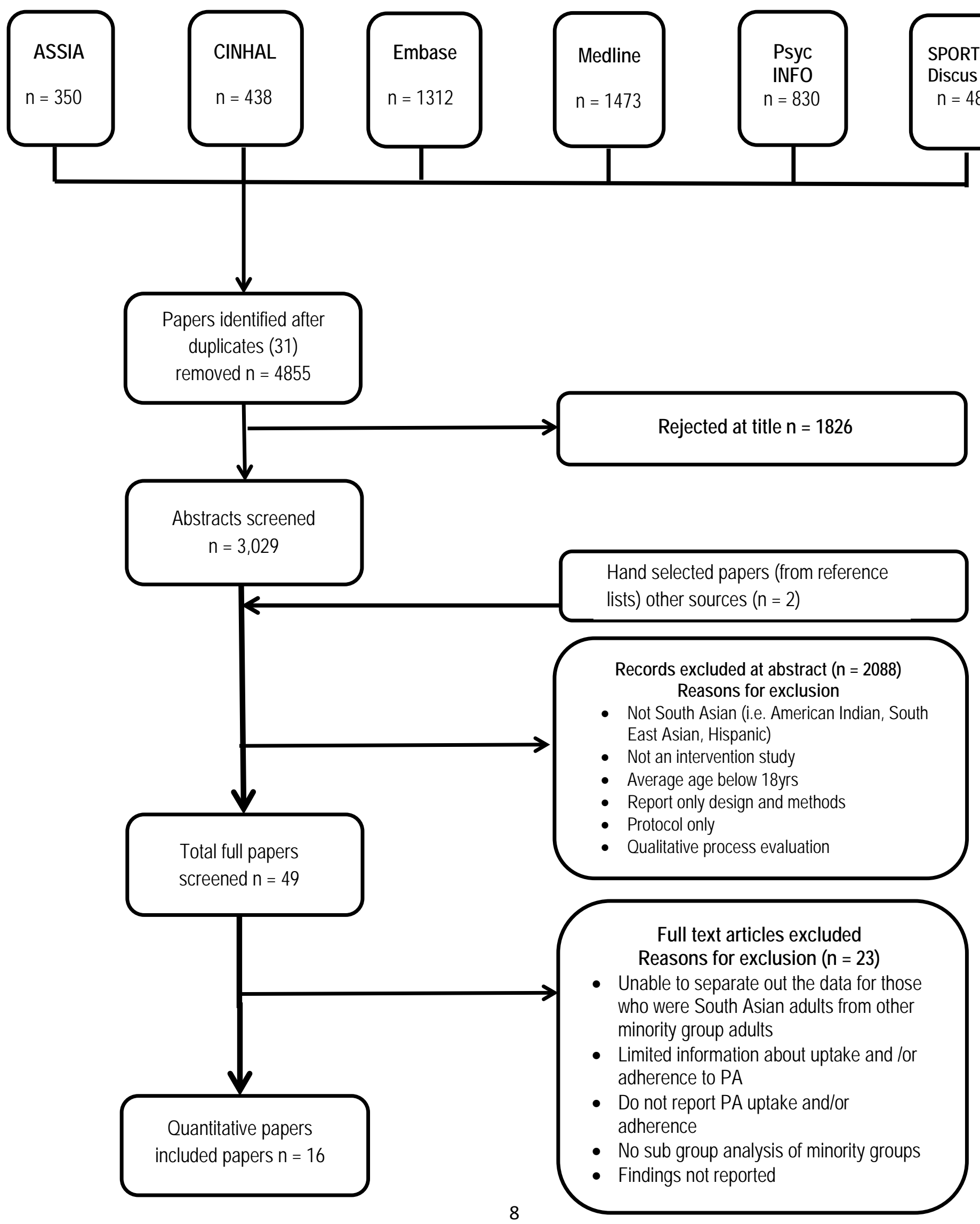


Appraisal of studies

All included studies/papers were of good or adequate quality (Table 1). The research design and reporting of the findings were not clear or well described in two thirds of the included studies/papers.

PA interventions

PA interventions targeting SA adults were related to:

(i) DM management/risk factor reduction as an end point in ten studies [38,39-41,43,45-49]. In these studies, PA was part of (a) an educational programme addressing DM self-management [39-41] or (b) a text based messaging service $[46,47,48,49]$; only one DM risk management study had PA as a primary outcome measure [43].

(ii) Healthy lifestyle: PA was part of a group based intervention using aerobic exercise, built on SA folk dance [42].

(iii) PA as a primary outcome measure: These interventions focussed on promoting PA uptake in two rural Indian villages [50]; increasing PA uptake and long-term adherence among Pakistani men (one study, two papers) $[37,38]$; increasing PA among SA immigrant women (from Indian, Sri Lankan, Bangladeshi, Nepal and Pakistani to Canada) through culturally tailored dance programmes [51] and a culturally tailored dance programme or standard gym-based exercise [44].

Intervention content

Development of the intervention. Four interventions (five papers) were developed with representatives from the target SA community using qualitative methods [36,37,39-42] or survey to assess health needs [42] (Table 2) to (i) explore PA expectations, preferences and potential 
barriers to undertaking the intervention [36,37] and (ii) to develop culturally and/or linguistically tailored programmes $[39,40,42]$. One intervention used feedback from Indian consumers [46] to develop culturally tailored text messages designed to motivate healthy behaviours.

Design theory of content. Six studies (seven papers) used psychological theories to inform/underpin their interventions $[36-40,42,47$ ) (Table 2): (i) Social Cognitive Theory $[36,37,38,42]$, (ii) theory of planned behaviour [42]; (iii) Trans Theoretical Model (TTM) [38,47]; (iv) self-efficacy $[39,40]$.

Six studies used a mixture of behavioural approaches [41,43,45-48] (Table 2): (i) goal setting and motivational interviewing [41]; (ii) social support and role modelling, self-monitoring, goal setting, feedback and reinforcement [43]; (iii) experiential methods [45]; (iv) personalised education and motivation [47]; (v) individually and/or culturally tailored mobile text messaging $[46,47,48]$.

<Table 2>

Cultural adaptations. 10 studies (11 papers) reported using some form of cultural adaptations [36-46,51] (Table 2): (i) community representatives used to ensure cultural appropriateness [36,37]; (ii) culturally adapted materials, messages and images to take into account of language, dress [38,39,40,42,50]; (iii) culturally and linguistically tailored group education sessions [39,40]; (iv) culture-specific dance $[42,44,51]$ - a) aerobic exercise built on SA folk dance [42]; b) Bhangra dancing [44], led by a female SA personal trainer at a local fitness center in one arm of the trial; c) Bollywood dance [51]; (v) women-only exercises [39,43] and culturally appropriate exercise during household work [41]; (vi) culturally tailored intervention programmes, including mobile text messaging [38-40,42,45,46];

Three further studies did not report on any specific cultural adaptations to their intervention [47-49]; these studies were conducted in India or Pakistan. 
Intervention delivery

Seven studies [38-40,42, 45,50-51] utilised participatory/social marketing approaches to promote/implement community-based PA intervention (see Table 2).

Five studies (six papers) used some form of target community involvement/resource to deliver the intervention [36,37,39,40,42,50] (Table 2). This included: a) community representatives and groups [50] to overcome barriers and facilitate program outcomes; b) festive gatherings (Melas)/community settings $[39,40,42,43]$ to incorporate culturally-salient activities like yoga, aerobic exercise and for convenience; c) Community Health Workers (CHWs) from targeted areas $[40,42]$; d) SA advisory board $[36,37,42]$ to plan and develop the intervention and review study materials and questionnaires to ensure they were culturally appropriate/meaningful; e) link workers and self-help groups to deliver the PA programme and provide a source of motivation to maintain PA amongst a rural Indian community [50].

Six studies reported using trained, bilingual staff or staff with same background i.e. gender, ethnicity for intervention delivery $[39,40,43,44,45,51]$ and one study reported using group classes based on language preference [42] (Table 2).

\section{Outcomes}

(i) Increase in PA. Six studies found significant increases in PA [36,37,39-40,45] using a range of assessment methods (Table 1). One study (two papers) used accelerometers [36,37]. Andersen et al [37] reported significant increases in PA levels (counts per minute (CPM)) in the intervention group compared to controls after the 5-month intervention $(p=0.01)$ and significant increases from baseline in the intervention group compared to controls $(p=0.02) 6$ months post intervention [36]. Additionally, the amount of moderate to vigorous PA increased more for the intervention group compared to controls at 6 month follow-up ( $p=0.003$ ), whilst amount of sedentary time decreased more in the intervention group compared to controls at 6 month follow up $(p=0.001)$ [36]. PA self-report

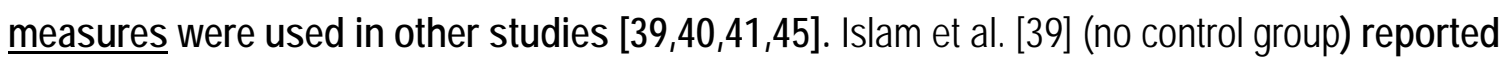
significant increases in PA from baseline to 12 months $(p<0.001)$ and Islam et al. [40] (control group included) reported significant increases in PA from baseline to 6 months $(p<0.01)$ compared to controls. Patel et al [45] found significant increases in PA between baseline and 
post-test at 12 weeks $(p<0.0005)$ and baseline and follow-up test at 24 weeks $(p=0.001)$ regardless of group to which they were assigned.

(ii) Increase in participation rates. Participation rates, as a success measure, were included in four studies [43,45,50,51]. In Subitha et al's [50] community-based PA intervention in a rural Indian Community, $54.6 \%$ (265/485 people) stated that they engaged in brisk walking $>4$ days a week; a further $32.2 \%$ (156/485 people) reported walking on 1-4 days per week during the 10-week intervention. Likewise, Vahabi and Damba [51] reported an average attendance of 85\% (range 77\%-96\%) during a twice weekly, 6-week Bollywood dance intervention. Similarly, Kandula et al. [43] reported 100\% retention rates at 3 and 6 months, with intervention participants attending 5 out of 6 sessions on average (range 1-6), for a culturally tailored exercise intervention for SA immigrant mothers with DM risk factors. Likewise, Lesser et al's [44] RCT of Bhangra dance or standard exercise program on visceral adipose tissue (VAT), reported average attendance of $78 \%+/-33 \%$ and $67 \%+/-25 \%$, respectively (of 36 delivered exercise classes). Similarly, Patel et al's [45] pre-test post-test study reported average weekly attendance of 7.4 out of 12 and $80 \%$ retention rates post-test (12 weeks) for the intervention group; control group retention rate was $83 \%$. However, using attendance as a proxy for actual PA does not confirm that those attending engaged in the activity.

(iii) PA self-efficacy and outcome expectancy. PA self-efficacy and outcome expectancy, as a measure of success, were reported in three studies [36,39,51]. Islam et al [39] reported increases in PA self-efficacy post-intervention, with all participants moderately or very confident that they could engage in regular PA at 12 months follow-up and 89\% of participants exercising at least several times a week compared to $52 \%$ at baseline (based on self-reported measures). Although self-efficacy did not differ between intervention and control groups in Andersen et al [36], they did note significantly higher outcome expectancy scores in the Pakistani men - intervention group (mean difference $=0.6 ; \mathrm{Cl}$ $=0.9$ to $0.2 ; P<0.01)$. Similarly, Vahabi and Damba [51] found that although there was a small increase in Outcome Expectations for Exercise score pre- and post-intervention, these changes were not significant.

(iv) PA as a secondary outcome measure. PA was a secondary outcome measure in 11 studies [38-41-43,45-49] (see Table 1). Significant increases in PA were found in two studies $[45,48]$. 
Patel et al. [45] observed a significant increase in PA at follow-up analysis between baseline and posttest at 12 weeks $(p<0.0005)$ and baseline and follow-up at 24 weeks $(p=0.001)$. Shahid et al. [48] found a significant increase in physically active patients at 4 months in the intervention group $(16.4 \%$ to $44.5 \% ; p<0.001)$, compared to the control group $(14.1 \%$ to $16.4 \% ; p=0.472)$.

\section{Discussion}

The aim of this review was to locate studies of interventions aimed at SA adults to increase PA and to identify to identify specific changes to their content and delivery mode for this population. An important finding was that culturally-adapted PA interventions tended to be more accepted by the target community. However, although some studies demonstrated improved PA uptake and/or adherence, this was not consistently evidenced across all included studies. Nevertheless, it supports, to some extent evidence from previous studies involving other ethnic/cultural groups [52] and systematic reviews [13,53], which suggest that cultural adaptations of interventions make them more accepted and effective.

Our reivew found several cultural adaptations/strategies that seem to improve PA uptake and/or adherence: (i) using participatory approaches to identify relevant cultural attitudes and norms to inform PA interventions; (ii) adapting content of intervention materials, images and messages to take into account language, dress and the target populations explanatory models to undertaking self-care behaviour (iii) using CHWs or ethnic/culture specific local facilitators/trained bilingual staff to overcome the barriers to intervention uptake; (iv) using community/neighbourhood facilities/places of worship; (v) including culture-specific dance.

Involving the target community in developing culturally appropriate interventions appears to be important in their acceptability, delivery and take-up. In particular, community-based participation in intervention planning, evaluation and research may help to produce culturally and linguistically tailored interventions that address core values, attitudes, beliefs and norms and encourage participation by ensuring recruitment and retention appropriately address community needs and barriers $[9,40,50,54]$. Likewise, undertaking prior qualitative work with the target community allows for identification of relevant cultural attitudes and norms to inform PA interventions [36,37]. Hence, studies included in this review highlight added benefits to using community-based approaches: (i) recruitment of participants and (ii) facilitating access to PA interventions. This is supported by the literature $[55,56]$. 
The site of intervention delivery, specifically the use of community/neighbourhood facilities and melas/religious festivals, appears to be important in terms of acceptability, take-up and adherence. Likewise, using culture-specific dance [60] may increase impact and sustainability of PA [57].

Although not specific to SA adults, a review by Davidson et al. [54] concurs overall with the above cultural adaptations, such as use of places of worship where minority groups congregate and use of culture specific dance. In our review, studies showed how Indian communities, not only embraced the dances, but also sustained this form of PA. Contrary, Davidson et al. [54] report how perceived relevance to adaptations for behaviour change to improve health is dependent on specific cultural priorities (p. 828). For example, adapted interventions for weight loss, such as portion control may not be acceptable in some cultures as being fat is seen as 'beautiful' [54].

Davidson et al. [54] also note that cultural adaptation improved usage of interventions for diet and PA to promote health among minority groups. The importance of context, that can either enhance or reduce the effectiveness of adaptations, was also highlighted. Davidson et al. [54] developed a typology of adaptation approaches through conducting seven systematic reviews and evaluating 107 studies. They argued that healthcare professionals need to include a particular minority group's cultural context into the adaptations for an intervention to work and be accepted [65]. This point may be the reason why some studies in our review did not show acceptance by minority populations even though cultural adaptation was undertaken. The findings of our review, therefore support Davidson et al's [54] notion that minority groups are not homogenous, rather they are heterogenic with their own cultural context. Hence adaptations need to be culturally-context specific.

It should be noted that our review included studies conducted inside and outside of SA countries, to capture as much data as possible due to the paucity of evidence in this field. Taking this approach provides a broad sense of what has been researched to support and promote PA among SA adults. Furthermore, it reflects the range of strategies, in terms of content and delivery mode, adopted to address PA among this population, regardless of where they are living. It is acknowledged that such wide-ranging inclusion criteria could be perceived 
as a limitation. However, it does add to the argument that the current evidence base is lacking around the topic of cultural adaptation, given that there were no clear distinctions in effectiveness in terms of studies that did and did not include such modifications. It should also be noted that studies conducted in SA countries tended to focus on diabetes, rather than considering PA as a general health behaviour, and on increasing PA levels among rural communities, highlighting a gap in knowledge that researchers can examine going forward.

\section{Conclusion}

Making cultural adaptations to PA interventions is a complex area. From the way the studies were designed, it was difficult to isolate the effects of cultural adaption. Nevertheless, making cultural adaptations shows promise and should be followed up with research using more robust designs following the MRC framework for developing complex interventions [61]. The evidence presented currently is not strong enough. However, it does not suggest that making adaptations is ineffective.

\section{Author statements \\ Ethical approval \\ None sought}

\section{Funding}

The review was funded as part of a research project by the NIHR Greater Manchester Collaboration for Leadership in Applied Health Research and Care (CLAHRC), RCF_R9_MH. The funding body had no input to the design of the study and collection, analysis, and interpretation of data or in writing the manuscript.

\section{Competing interests}

None declared. 


\section{Acknowledgements}

We would like to thank Adrine Woodham for her part in undertaking some of the earlier database searches. 


\section{References}

1 Department of Health. Physical activity guideline for adults (19-64 years). London: DH, 2011.

2 World Health Organization. Global recommendations on physical activity for health. Geneva: WHO, 2010.

3 Hosper K, Nierkens V, Nicolaou M, Stronks K. Behavioural risk factors in two generations of nonWestern migrants: do trends converge towards the host population? Eur J Epidemiol, 2007;22:163-172.

4 NHS Information Centre. Health Survey for England 2004: The Health of Minority Ethnic Groups. London: Information Centre, 2006.

5 Gill PS, Kai J, Bhopal RS, Wild S. Health Care Needs Assessment of Black and Minority Ethnic Groups. In: Stevens A, Raftery J, Mant J, Simpson S (eds). The Epidemiologically Based Health Needs Assessment Reviews. London: Radcliffe, 2007.

6 Sproston K, J Mindell (eds). Health Survey for England 2004: The Health of Minority Ethnic Groups. London: Information Centre; 2006.

7 Ghouri NG, Purves D, McConnachie A, Wilson J, Gill JMR. \& Sattar N. Lower cardiorespiratory fitness contributes to increased insulin resistance and fasting glycaemia in middle-aged South Asian compared with European men living in the UK. Diabetologia, 2013; 56 (10): 2238-2249.

8 European Commission. Commission staff working document. Report on health inequalities in the European Union. Brussels: EC; 2013. Available

at: http://ec.europa.eu/health/social determinants/docs/report healthinequalities swd 2013328 en.p df [accessed 21st November 2017].

9 Department of Health and Human Services (DHHS). HHS Action Plan to Reduce Racial and Ethnic Health Disparities. Office of Minority Health, 2011. Available

at: http://minorityhealth.hhs.gov/npa/files/Plans/HHS/HHS Plan complete.pdf [accessed 21 1 st

November 2017].

10 Marmot M. Fair Society, Healthy Lives. Strategic Review of Health Inequalities in England post-2010 (the Marmot review). London: The Marmot Review, 2010.

11 Department of Health. Start active, stay active. A report on physical activity for health from the four home countries' Chief Medical Officers. London: Crown Copyright, 2011.

12 Netto G, Bhopal R, Lederle N, Khatoon J, Jackson A. How can health promotion interventions be adapted for minority ethnic communities? Five principles for guiding the development of behavioural interventions. Health Promot Int, 2010; 25:248-257. 
13 Lui JJ, Davidson E, Bhopal RS et al. Adapting health promotion interventions to meet the needs of ethnic minority groups: mixed-methods evidence synthesis. Health Tech Assess, 2012;16: 44. doi: http://dx.doi.org/10.3310/hta16440

14 King AC, Sallis JF. Why and how to improve physical activity promotion: Lessons from behavioural science and related fields. Prev Med, 2009;49:286-288.

15 Barrera M Jr, Castro FG, Steiker LK. A Critical Analysis of Approaches to the Development of Preventive Interventions for Subcultural Groups. Am J Community Psychol, 2011;48:439-454.

16 Kupfer KL, Alvarado R, Smith P, Bellamy, N. Cultural sensitivity and adaptation in family-based prevention interventions. Prev Sci, 2002;3:241-246.

17 Kleinman A, Eisenberg L, Good B. Culture, illness, and care: clinical lessons from anthropologic and cross-cultural research. Ann Intern Med, 1978; 88 (2): 251-8.

18. Kandula NR., Khurana NR., Makoul G, Glass S \& Baker DW (2012) A Community and CultureCentered Approach to Developing Effective Cardiovascular Health Messages. J Gen Intern Med 27(10):1308-16.

19 NICE \& WHO Europe. Health systems and health-related behaviour change: a review of primary and secondary evidence, 2011. Available at: https://www.nice.org.uk/media/default/About/what-wedo/NICE-guidance/NICE-guidelines/Public-health-guidelines/Additional-publications/Special-reporthealth-systems-and-health-related-behaviour-change.pdf [accessed 21st November 2017].

20 Barrera M Jr., Castro F.G., Stryker LA, Toobert DJ. Cultural Adaptations of Behavioral Health Interventions: A Progress Report. Am J Community Psychol, 2013;81:196-205.

21 Department of Health. No Patient Left Behind: How Can We Ensure World Class Primary Care for Black and Minority Ethnic People? London: HMSO, 2008.

22 Department of Health. Healthy lives, healthy people: our strategy for public health in England. London: Stationery Office, 2010.

23 Horne M, Tierney S. What are the barriers and facilitators to exercise and physical activity uptake and adherence among South Asian older adults: A systematic review of qualitative studies. Prev Med, 2012;55:276-284.

24 Horne M, Skelton D, Speed S, Todd C. Attitudes and beliefs to the uptake and maintenance of physical activity among community dwelling South Asian 60-70 year-olds: A qualitative study. Public Health, 2012;126:417-423.

25 Babakus WS, Thompson JL. Physical activity among South Asian women: a systematic, mixedmethods review. Int J Behav Nutr Phys Activ, 2012;9:150. 
26 Ranasinghe $C D$, Ranasinghe $P$, Jayawardena $R$, Misra A. Physical activity patterns among SouthAsian adults: a systematic review. Int J Behav Nutr Phys Activ, 2013;10:116.

27 Chapman J, Qureshi N, Kai J. Effectiveness of physical activity and dietary interventions in South Asian populations: A systematic review. Br J Gen Pract, 2013;63: e-104-e-114.

28 BrownT, Smith S, Bhopal R, Kasim A \& Summerbell C. Diet and physical activity interventions to prevent or treat obesity in South Asian children and adults: A systematic review and meta-analysis. International Journal of Environmental Research and Public Health, 2015; 12: 566-594.

29 CRD. Systematic Reviews. CRD's guidance for undertaking reviews in health care. University of York, York: CRD, 2009.

30 Higgins JPT, Green S (eds). Cochrane Handbook for Systematic Reviews of Interventions, Version 5.1.0. The Cochrane Collaboration, 2011. Available at: http://handbook-5-1.cochrane.org/ [accessed 21 $1^{\text {st }}$ Novemeber 2017].

31 Moher D, Liberati A, Tetzlaff J, Altman DG. PRISMA Group. Preferred reporting items for systematic reviews and meta-analyses: the PRISMA statement. PLoS Med, 2009;6:e1000097. Epub 2009 Jul 21. PubMed PMID: 19621072.

32 Caspersen CJ, Powell KE, Christenson GM. Physical activity, exercise, and physical fitness: definitions and distinctions for health-related research. Public Health Rep, 1985; 100(2):126-31.

33 Scottish Intercollegiate Guidelines Network. SIGN 50. A Guideline Developer's Handbook. Edinburgh: SIGN, 2001.

34 Stiggelbout M, Hopman-Rock M, Van Mechelen W. Entry correlates and motivations of older adults participating in organized exercise programs. J Aging Phys Activ, 2008;16: 342-354.

35 Petticrew P, Roberts H. Systematic reviews in the social sciences: a practical guide. Oxford: Blackwell Publishing; 2006.

36 Andersen E, Burton NW, Andersen SA. Physical activity levels six months after a randomised controlled physical activity intervention for Pakistani immigrant men living in Norway. Int J Behav Nutr Phys Activ, 2012; 9:47.

37 Andersen E, Høstmark AT, Holme I, Andersen SA. Intervention effects on Physical Activity and insulin levels in men of Pakistani origin living in Oslo: A Randomised Controlled Trial. J Immigr Minor Health, 2013;15:101-110.

38 Bhopal R, Douglas A, Wallia S, Forbes JF, Lean ME et al. Effect of a lifestyle intervention on weight change in South Asian individuals in the UK at high risk of type 2 diabetes: a family-cluster randomised controlled trial. Lancet, 2014; 2: 210-217.

39 Islam NS, Wyatt LC, Patel SD et al. Evaluation of a community health worker pilot intervention to improve diabetes management in Bangladeshi immigrants with type 2 diabetes in New York City. Diabetes Educat, 2013;39:478-493. 
40 Islam NS, Zanowiak JM, Wyatt LC et al. Diabetes Prevention in the New York City Sikh Asian Indian Community: A Pilot Study. Int. J. Environ. Res. Public Health, 2014;11:5462-5486.

41 Jayasuriya $R$, Pinidiyapathirage $M J$, Jayawardena $R$, Kasturiratne $A$, de Zoysa $P$, Godamunne $P$, Gamage S, Wickremasinghe AR. Translational research for Diabetes Self-Management in Sri Lanka: A randomized controlled trial. Prim Care Diabetes, 2015; 9:338-345.

42 Kandula NR., Swapna D., De Chavez PJ., Bharucha H., Patel Y., Sequil P., Kumar S, Baker DW., Spring B \& Siddique J (2015) Translating a heart disease lifestyle intervention into the community: the

South Asian Heart Lifestyle Intervention (SAHELI) study; a randomized control trial. BMC Public Health, 5:1064.

43 Kandula NR., Swapna D., De Chavez PJ., Marquez DX., Bharucha H., Mammen SM., Dunaif A., Ackermann RT., Kumar S \& Siddique J (2016) An Exercise Intervention for South Asian Mothers with Risk Factors for Diabetes. Translational Journal of the ACSM, 1 (6): 52-59.

44 Lesser I., Singer J., Hoogbruin A., Makey DC., Katzmarzyk PT., Sohul P., Leipsic J \& Lear S. (2016) Effectiveness of Exercise on Visceral Adipose Tissue in Older South Asian Women. Med Sci Sports Exerc, 2016; 48 (7):1371-1378.

45 Patel RM., Misra R., Raj S \& Balasubramanya A (2017) Effectiveness of a Group-Based Culturally Tailored Lifestyle Intervention Program on Changes in Risk Factors for Type 2 Diabetes among Asian Indians in the United States. Journal of Diabetes Research, doi.org/10.1155/2017/2751980.

46 Pfammatter A, Spring B, Saligram N, Dave R, Gowda A, Blais L, Arora M, Ranjani H, Ganda O, Hedeker D, Reddy S, Ramalingam S. mHealth Intervention to Improve Diabetes Risk Behaviors in India: A Prospective, Parallel Group Cohort Study. J Med Internet Res, 2016; 18 (8):e207.

47 Ramachandran A, Snehalatha C, Ram J, Selvan S, Simon M, Nanditha A, Shetty AS, Godsland IF, Chaturvedi N, Majeed A, Oliver N, Toumazou C, Alberti KG, Johnston DG. Eff ectiveness of mobile phone messaging in prevention of type 2 diabetes by lifestyle modifi cation in men in India: a prospective, parallel-group, randomised controlled trial. Lancet Diabetes Endocrinol, 2013; 1: 191-98.

48 Shahid M, Mahar SA, Shaikh S, Shaikh Z. Mobile Phone Intervention to Improve Diabetes Care in Rural Areas of Pakistan: A Randomized Controlled Trial. Journal of the College of Physicians and Surgeons Pakistan 2015; 25 (3): 166-171.

49 Shetty AS, Chamukuttan S, Nanditha A et al. Reinforcement of adherence to prescription recommendations in Asian Indian diabetes patients using Short Message Service (SMS)- A pilot study. JAPI, 2011;59:711-714.

50 Subitha L, Soudarssanane MB, Murugesan R. Community-based physical activity intervention using principles of social marketing: A demonstration project in southern India. Natl. Med. J. India, 2013; 26(1):12-17. 
51 Vahabi M, Damba C. A Feasibility Study of a culturally and gender-specific Dance to promote physical activity for South Asian immigrant women in the greater Toronto area. Women's Health Iss, 2015;25(1):79-87.

52 Keyserling TC, Samuel-Hodge CD, Ammerman AS et al. A randomized trial of an intervention to improve selfcare behaviors of African-American women with type 2 diabetes: impact on physical activity. Diabetes Care, 2002;25:1576-1583.

53 Skaff MM, Chesla CA, Mycue VD, Fisher L. Lessons in cultural competence: Adapting research methodology for Latino participants. J Community Psychol, 2002;30:305-323.

54 Davidson EM, Liu JJ, Bhopal R, White M, Wabnitz C, Sheikh A. Behavior Change interventions to improve the health of racial and ethnic minority populations: A tool kit of adaptation approaches. Milbank Quart, 2013;91:811-851.

55 Resnikow K, Soler R, Braithwait RL, Ahluwalia JS, Butler J. Cultural sensitivity in substance abuse prevention. J Community Psychol, 2000; 28:271-290.

56 Netto G, Bhopal R, Jackson, A., Lederle N, Jackson A. Health promotion and prevention interventions in Pakistani, Chinese and Indian communities related to CVD and cancer: a review of the published evidence in the UK, other parts of Europe and the United States. NHS Health Scotland, 2010. Available at: www.equalitiesinhealth.org/doc/race/Review-of-health-interventions.doc Accessed 21st November 2017.

57 Conn VS, Chan K, Banks J, Ruppar TM, Scharff J. Cultural relevance of physical activity intervention research with underrepresented populations. Int Q Community Health Educ, 2013; 34(4):391-414.

58 Eng E, Parker E, Harlan C. Lay health advisor intervention strategies: A continuum from natural helping to paraprofessional helping. Health Euduc Behav, 1997;24:413-417.

59 Perry HB, Zulliger R, Rogers MM. Community health workers in low-, middle-, and high-income countries: An overview of their history, recent evolution, and current effectiveness. Annu Rev Publ Health, 2014;35: 399-421

60 Jain S, Brown D. Cultural dance: An opportunity to encourage physical activity and health in communities. Am J Health Educ, 2001;32:216-222.

61 Campbell NC, Murray, E, Darbyshire J, Emery J, Farmer A, Griffiths F, et al., 2007. Designing and evaluating complex interventions to health care. British Medical Journal, 2017; 334: 455-459.

62 Wallia S, Bhopal RS, Douglas A, Bhopal R, Sharma A, Hutchinson A, Murray G, Gill J, Sattar N, Lawton J, Tuomilehto J, McNight J, Forbes J, Lean M, Sheikh A. Culturally adapting the prevention of diabetes and obesity in South Asians (PODOSA) trial. Health Promotion International, 2014; 29: 768769 . 